\title{
No prophecies for the superstitious
}

\section{There is no way of telling what discoveries lie ahead, but some of the baggage of conundrums left unanswered in 1987 may be instructive.}

SUPERSTITIOUS earlier generations used to mark new years with the publication of almanacs which, by judiciously blending near-certainty (the time of sunset) with speculation ("the King will die"), gave often-wide currency to unsubstantiable, but also irrefutable, soothsayings. In the nature of things, predicting the future course of discovery, the process of learning the unexpected, is a contradiction in terms. What follows, therefore, is not an almanac in the old sense, but a listing of some of the interesting questions left unanswered at the end of 1987.

The most clamant of these questions is also the most puzzling - that of knowing the mechanism of superconductivity in the ceramic 'high-temperature' superconductors. It is just 14 months since Müller and Bednorz announced the measurement of superconductivity at above $30 \mathrm{~K}$ in lanthanum-barium-copper oxide, and less than a year since Chu and his colleagues at the University of Houston described superconductivity at $90 \mathrm{~K}$ in an yttrium analogue.

Now it seems that there may be two classes of ceramic superconductors, with transition temperatures of 30 and $90 \mathrm{~K}$, respectively. Certainly, those finding evidence for superconductivity nearer to room temperature have mostly been compelled, with disappointment, to account for their observations in terms of surface phenomena or as otherwise exceptional happenings (which are none the less intriguing on that account).

For the time being, much the same is true of the explanations of the new phenomena on offer. The standard theory of conventional superconductors is itself notoriously incapable of predicting transition temperatures accurately, but nobody should be surprised at that, given the complexity of all attempts to calculate phase transitions for cooperative systems.

Part of the difficulty with the new materials is that the interaction between electrons must entail exchange interactions between copper atoms through the intermediary of intervening oxygens (whence the suspicion that Anderson's resonating valence-bond theory must be on the right lines); the snag is the formidable complexity of integrating all this with the thermodynamics of a vibrating lattice, but there must surely be some break in the problem in the year ahead.

There can be few theoretical issues whose resolution would bring such important prizes. While empirical methods have done so little to provide materials with higher transition temperatures, understanding seems the surest way forward. Meanwhile, there are many who will recall Professor V.L. Ginzburg's powerful case (at the Nature conference in November) that even liquid-nitrogen superconductors will be a great boon; letting the nitrogen boil off is a reasonable option.

The excitement over superconductivity is sometimes said to have been the best thing to have happened to solid-state physics for 20 years, but that is of course either a canard or an illusion. For quite different reasons, but in different ways, the field has been booming for several years, as shown by the now almost routine measurements of surface structure (with scanning tunnelling microscopes as well as diffraction techniques). What seems to be happening is rapid progress towards a state of affairs in which atomic structures are directly observable, by one technique or another. Can it be long before atom-byatom fabrication is also possible?

Precision spectroscopy is similarly full of promise. Quite astonishing things are possible. In the latest issue of Physical Review Letters, for example, F. Diedrich et al. from the Max Planck Institute of quantum optics at Garching describe the measurement by spectroscopic techniques of the 'phase transition' between 'solid' and 'gaseous' clusters of laser-cooled magnesium ions held in an electromagnetic trap (there is a photograph of the fluorescent images of seven such ions held in an asymmetric hexagon); another West German group (J. Neukammer et al., most from the Free University of Berlin) has measured barium atoms with an outer electron in a state whose principal quantum number exceeds 500 (which are sensitive measures of feeble electric fields); and F. de Martini et al. (from the University of Rome) have been able to demonstrate the prolongation of the lifetime of excited dye molecules in a Fabry-Perot optical cavity, thereby showing in the most direct fashion possible the coupling of atoms (or molecules, in this case) to the states of the radiation field in which they are immersed. Sooner rather than later, the precision of the new spectroscopy will yield new frequency standards, but there will be greater benefits along the way.

On the grander questions (What is matter? Where did it come from?) there is at least some hope that the question of the 'fifth force' (the short-range accompaniment of gravity) will be settled one way or another during 1988. High-energy physicists appear content that the 'top' quark exists, although confirmation must probably await the next generation of accelerators. Meanwhile, a Japanese group working with the new KEK electroncollider seems to have gone further than others in ruling out a fourth generation of leptons (after electrons, muons and tauons).

One setback for astronomers and for the rest of us is that the Hubble Space Telescope, potentially the most valuable of all Earth satellites so far, is still waiting on the ground in California for a now further delayed shuttle launch, while the Voyager spacecraft will not reach Neptune until next year.

Has molecular biology by comparison become flat, almost dull? That the question can even be asked about what has been the cutting edge of biology for the past 30 years points to what seems an important transformation of the field. That too is a canard, but questions such as the mechanism of the differentiation of cells, or tissues, seem almost as obscure now as a decade ago, chiefly because research has so far uncovered mostly the complexities.

In any case, the flood of discoveries in the past two years about the genetic basis of inherited diseases as different as muscular dystrophy and manic-depressive illness is certain to continue, and to yield an understanding of mechanisms in some cases. Indeed, that field, the unravelling of the intricacies of the immune system (with its bearing, among other things, on AIDS, acquired immune deficiency syndrome) and the demands of biotechnology are a reminder that, even after the huge growth of molecular biology in the past decade, there is now much more valuable work to do than there are skilled people to undertake it.

Meanwhile, it is natural but exciting that interest in neural networks - a way of modelling nervous systems - should have succeeded in capturing so much interest from a wide and interdisciplinary group of people, from molecular biologists to electrical engineers. Those insisting on some kind of a prediction for 1988 might take a chance of something in that field. But what?

John Maddox 